\title{
mTORC1/2 Inhibitor Served as a More Ideal Agent Against the Growth of Mouse Lymphocytic Leukemia Both In Vitro and In Vivo
}

\author{
HUI-FEN LIAO ${ }^{1}$, YOU-ZHU LIN ${ }^{1}$, CHIH-CHIA YU ${ }^{2}$, TZONG-SHYUAN TAI ${ }^{3}$, \\ SHIH-KAI HUNG ${ }^{2}$, CHING-CHIEH YANG ${ }^{4,5,6}$ and YU-CHIEH SU ${ }^{7,8}$ \\ ${ }^{1}$ Department of Biochemical Science and Technology, National Chiayi University, Chiayi, Taiwan, R.O.C.; \\ ${ }^{2}$ Department of Radiation Oncology, Dalin Tzu Chi Hospital, \\ Buddhist Tzu Chi Medical Foundation, Chiayi, Taiwan, R.O.C.; \\ ${ }^{3}$ Department of Medical Research, E-Da Hospital, Kaohsiung, Taiwan, R.O.C.; \\ ${ }^{4}$ Department of Radiation Oncology, Chi-Mei Medical Center, Tainan, Taiwan, R.O.C.; \\ ${ }^{5}$ Institute of Biomedical Sciences, National Sun Yat-Sen University, Kaohsiung, Taiwan, R.O.C.; \\ ${ }^{6}$ Department of Pharmacy, Chia-Nan University of Pharmacy and Science, Tainan, Taiwan, R.O.C.; \\ ${ }^{7}$ Division of Hematology-Oncology, Department of Internal Medicine, \\ Kaohsiung Medical University Hospital, Kaohsiung, Taiwan, R.O.C.; \\ ${ }^{8}$ Faculty of Medicine, Kaohsiung Medical University, Kaohsiung, Taiwan, R.O.C.
}

\begin{abstract}
Background/Aim: Chronic lymphocytic leukemia (CLL) still remains an incurable disease as the cells evade apoptosis, which is an obstacle for current therapeutic approaches. Therefore, our aim was to identify an ideal target of leukemic cell growth for developing inhibitors. Materials and Methods: Mouse lymphocytic leukemia cell line L1210, human Toledo cells and a DBA/2 mouse graft model were used to analyze the activity of dual mTORC1/2 inhibitor AZD2014s. Western blotting and flow cytometry were performed to determine the mechanism. Results: AZD2014 inhibited L1210 and human Toledo cell proliferation. Treatment with AZD2014 reduced the phosphorylation levels of S6K1 and $4 E B P 1$ and the protein levels of Rictor, a component of the mTORC2 pathway. AZD2014 induced cell cycle arrest at the $G_{0}-G_{1}$ phase by reducing the expression of cyclin DI and CDK4. Oral administration of AZD2014 significantly inhibited the growth of L1210 cell grafts in DBA/2 mice. Conclusion: The mTORC1/2
\end{abstract}

Correspondence to: Yu-Chieh Su, Division of Hematology and Oncology, Department of Internal Medicine, Kaohsiung Medical University Hospital, 807, No 100, Tzyou 1st road, Kaohsiung, Taiwan, R.O.C. Tel: +886 73121101, Fax: +886 73212606, e-mail: hepatoma@gmail.com and Ching-Chieh Yang, Department of Radiation Oncology, Chi-Mei Medical Center, 701, No. 901 Zhonghua Rd., Yung Kang district, Tainan, Taiwan, R.O.C. Tel: +886 6281281153501, Fax: +886 62820049, e-mail: cleanclear0905@gmail.com

Key Words: Chronic lymphocytic leukemia, mammalian target of rapamycin, mTORC1/2, AZD2014. inhibitor may be a better therapeutic agent compared to PI3K/mTORC1 inhibitors for treating patients with CLL.

Chronic lymphocytic leukemia (CLL) occurs due to the proliferation and accumulation of B lymphocytes in the lymph nodes (1). CLL can be categorized into two major types: i) those with a somatically mutated immunoglobulin heavy variable gene and ii) those without this mutation, which serves as a prognostic marker for predicting patient survival outcome (2). The phosphatidylinositol 3-kinase (PI3K) inhibitor, idelalisib, and Bruton's tyrosine kinase (BTK) inhibitor, ibrutinib, have been demonstrated to exhibit a promising response in patients with relapsed or refractory CCL and without severe side-effects (3). Nevertheless, such treatments would induce prolonged lymphocytosis, which has been indicated to increase the probability of accumulating resistance-inducing mutations. Mutation in BTK or its downstream kinase has been detected in patients, causing resistance to ibrutinib (3). Therefore, there is a need to develop novel inhibitors or supplements for CLL treatment.

Several studies have been recently conducted aiming to develop drugs for CLL treatment that target other kinases of the PI3K family (4). The mammalian target of rapamycin (mTOR) is one of the major downstream kinases of the PI3k/AKT signaling pathway that regulates cellular proliferation and apoptosis (5). mTOR exists in two complexes, mTOR complex 1 (mTORC1) and 2 (mTORC2). When various factors bind to the cell surface tyrosine kinase receptors, mTORC1 (Raptor) is activated by phosphorylated 
AKT, promoting translation through the phosphorylation of the p70 S6 kinase (S6K), the S6 ribosome protein (S6), and the eukaryotic initiation factor 4E-binding protein 1 (4EBP1) $(6,7)$. Activated mTORC2 (Rictor) phosphorylates AKT, which is an important event for cell survival (8). Aberrant activation of mTORC1 is a common phenomenon observed in human cancer, which suggests that mTORC1 can be used as a target for developing inhibitors against various cancers (9). Nevertheless, inhibition of mTORC1 by rapamycin failed to induce cancer cell apoptosis in either cycling or quiescent cells (10). Remarkably, the dual inhibition of PI3K/mTORC1 or mTORC1/2 exhibited more potent efficacy against cancer growth in a wide range of malignancies.

This study investigated the anticancer potential of the clinical dual mTORC1/2 kinase inhibitor vistusertib (AZD2014) and the mTORC1 inhibitor (RAD001) in mouse leukemia L1210 cells and human Toledo cells. We further investigated the anticancer mechanism of AZD2014 and RAD001 in L1210 cells. To further evaluate and compare the anticancer activity of AZD2014 in vivo with the dual PI3K and mTORC1 inhibitor (BEZ235), we performed a L1210 leukemia graft model in DBA/2 mice.

\section{Materials and Methods}

Ethics statement. The male DBA/2 mice were obtained from BioLasco Taiwan Co., Ltd (Taipei, Taiwan). The experimental protocol was approved by the Animal Research committee of Kaohsiung Medical University of Taiwan under the guidance of the Public Health Service (PHS) policy on Human Care and Use of Laboratory Animals.

Cell lines and reagents. The parental mouse leukemia L1210 cells (ATCC, Rockville, MD, USA) and the human peripheral blood Toledo cell line (human diffuse large B-cell lymphomas cell line, ATCC, Rockville, MD, USA) were cultured in RPMI 1640 culture medium (Gibco Laborato- ties, Grand Island, NY, USA) supplemented with $10 \%$ fetal bovine serum and $1 \%$ antibioticantimycotic. The mTORC1 inhibitor, RAD001, and the mTORC1 inhibitor, BEZ235, the PI3K inhibitor, BYL719, and the pan-PI3K inhibitor, BKM120, were obtained from Novartis Pharmaceuticals Corporation (East Hanover, NJ, USA). The inhibitor AZD2014 was obtained from AstraZeneca (London, UK).

Antileukemic activity in vivo. Male DBA/2 mice aged 6 weeks were used in the present study. A total of 24 mice were randomly divided into 3 groups. All mice subcutaneously received L1210 cells for 5 days. The mice were given oral treatment daily with: i) vehicle, ii) AZD2014 (11.5 mg/kg), or iii) BEZ235 $(29 \mathrm{mg} / \mathrm{kg})$ from day 6 to day 10 . Tumor volumes were measured every day using calipers and were calculated using the formula $\left(\mathrm{L} \times \mathrm{W}^{2}\right) / 2$, where $\mathrm{L}$ and $\mathrm{W}$ indicate the length and width, respectively. All mice were sacrificed at day 14 , and the tumors were excised.

Cytotoxicity assay. Cytotoxicity was assessed using the colorimetric 3-(4,5-dimethylthiazol-2-yl)-5-(3-carboxymethoxyphenyl)-2-(4-sulfophenyl)-2H-tetrazolium (MTS) assay (Promega, Madison, WI, USA) according to the manufacturer's instructions. Briefly, the cells were seeded into a 96-well plate with or without serial dilution of AZD2014 and RAD001. Cell viability was determined after 48 hours at $37^{\circ} \mathrm{C}$, and relative cytotoxicity was quantified by a $550 \mathrm{Bio}-\mathrm{Rad}$ plate reader (Bio-Rad, Hertfordshire, UK) at an absorbance of $490 \mathrm{~nm}$.

Western blotting. L1210 cells were seeded into a 6-well plate at a density of $8 \times 10^{5}$ cells/well. After 24 hours of incubation, the cells were treated with $200 \mathrm{nM}$ AZD2014 or $100 \mathrm{nM}$ RAD001 for 48 hours. The cells were resuspended in RIPA lysis buffer (Sigma-Aldrich Chemical Company) and the soluble lysate was determined using the Bio-Rad protein assay kit (Hercules, CA, USA). An equal amount of protein was loaded onto 10\% SDS-polyamide gel electrophoresis and then transferred to PVDF membranes. The protein of interest was detected using specific antibodies against phospho-mTOR (Ser2448), Rictor, phospho-AKT (Ser473), phospho-S6 (Ser235/236), phospho4EBP1(Ser65), eIF4E, CyclinD1, and CDK4 (Cell Signaling Technology, Beverly, MA, USA). The blotting signal was developed using a chemiluminescence (ECL) detection kit (Merck, Darmstadt, Germany). The relative blot intensities were quantified by densitometric scanning. The $\beta$-actin served as an equal loading control.

Cell cycle analysis. L1210 cells were treated with AZD2014 or RAD001 for 48 hours and were then stained with DAPI (Cell Signaling Technology, Beverly, MA, USA). The effects of the inhibitors on cell cycle were evaluated using Nucleo Counter NC3000 (ChemoMetec, Allerod, Denmark).

Statistical analysis. Data are expressed as mean \pm standard deviation. Each experiment was performed as three independent experiments. Significance of the data was calculated using Student's $t$-test. A $p$ Value of $<0.05$ was considered for statistical significance.

\section{Results}

AZD2014 and RAD001 reduce the viability of mouse L1210 cells and human Toledo cells. To investigate the inhibitory effects of the mTORC1/2 inhibitor AZD2014 and the mTORC1 inhibitor RAD001 on tumor growth, we performed cell-based experiments using mouse leukemia L1210 cells and human Toledo cells. First, L1210 cells were treated with AZD2014 and RAD001 at the indicated concentrations for 48 hours. The results of the cell viability assay revealed that AZD2014 and RAD001 significantly reduce the cell growth (Figure 1A), with AZD2014 showing a better anticancer effect on L1210 cells. Similarly, AZD2014 and RAD001 reduce the viability of human Toledo cells in a dose-dependent manner (Figure 1B). Then, we investigated the effect of PI3K inhibitor on leukemia, for which human Toledo cells were treated using either the PI3K inhibitor BYL719 or the pan-PI3K inhibitor BKM120 at the indicated concentrations for 48 hours. The results showed that BKM120, but not BYL719, dosedependently reduce the cell viability (Figure 1C and D).

AZD2014 and RAD001 downregulate the levels of mTORC1 signaling and cell cycle arrest. To evaluate the anticancer mechanism of AZD2014 and RAD001, L1210 cells were treated with the inhibitors at the indicated concentrations for 

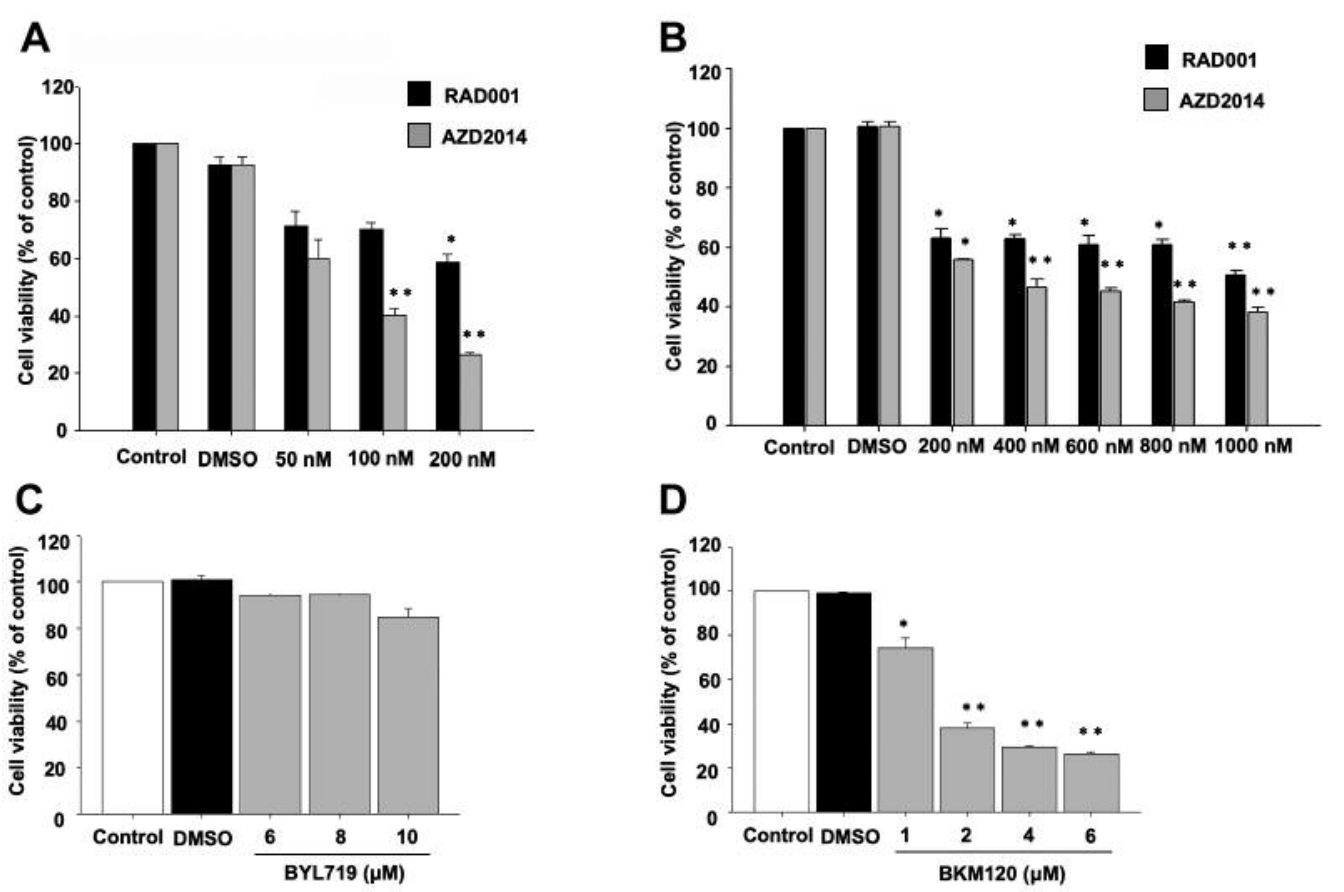

Figure 1. AZD2014, RAD001, and BKM120 reduced the viability of L1210 and human Toledo cells. L1210 cells were treated with AZD2014 or RAD001 at concentrations of: i) 50, ii) 100, and iii) $200 \mathrm{nM}$. Toledo cells were treated with AZD2014 or RAD001 at concentrations of: i) 200, ii) 400, iii) 600, iv) 800, and v) 1,000 nM for $48 \mathrm{~h}$. Toledo cells were treated with either BYL719 at concentrations of: i) 6, ii) 8, and iii) $10 \mu \mathrm{M}$ or with BKM120 at concentrations of: i) 1, ii) 2, iii) 4, and iv) $6 \mu \mathrm{M}$ for 48 hours. Data are indicative of at least three independent experiments, with each measurement performed as a triplicate of three independent experiments. Error bars are expressed as a mean $\pm S D\left({ }^{*} p<0.05, * * p<0.01\right)$.

48 hous. The western blotting results showed that AZD2014 significantly reduce the phosphorylation of AKT, mTOR, Rictor, S6, and 4EBP1, indicating that AZD2014 effectively block AKT/mTOR signaling (Figure 2). Comparatively, RAD001 significantly reduce the phosphorylation of AKT, Rictor, S6, and 4EBP1, but not of the mTOR activity (Figure 2). These results indicate that AZD2014 exhibits stronger inhibitory activity on the AKT/mTOR signaling pathway in L1210 cells compared to RAD001.

Previous research has reported that the mTOR signaling pathway positively controls cell proliferation and cell cycle progression by regulating S6K and 4EBP1 (11). Based on the abovementioned results, we further investigated the effect of AZD2014 and RAD001 on cell cycle progression in L1210 cells. The results of flow cytometry revealed that treatment with both inhibitors results in a significant increase in the proportion of L1210 cells in the G1 phase (Figure 3A). We also observed that AZD2014 significantly reduce the expression of cyclin D1 and CDK4, whereas RAD001 reduces only cyclin D1 expression (Figure 3B). These results indicate that the inhibitors induce G1 arrest in L1210 cells.

AZD2014 and BEZ235 effectively inhibit the growth of L1210 cell grafts in DBA/2 mice. To evaluate and compare the anticancer effects between AZD2014 and the PI3K/mTORC1 inhibitor BEZ235 in vivo, DBA/2 mice bearing the L1210 cell grafts were treated daily with: i) vehicle, ii) AZD2014, or iii) BEZ235 for 5 consecutive days from day 6 (Figure 4A). Their body weight and tumor volume were recorded daily. The results of the graft assay showed that the administration of AZD2014 and BEZ235 significantly reduces the tumor growth without decreasing the body weight of mice (Figure 4B and C). Mice in the AZD2014-treated group showed highest decrease in tumor volume in the L1210 cell graft model on day 7 following treatment with the inhibitor $\left(151.67 \pm 73.3 \mathrm{~mm}^{3}\right)$, as compared to the tumor volume of the BEZ235-treated group $\left(297.83 \pm 85.5 \mathrm{~mm}^{3}\right)$ or the vehicle-treated group $\left(1550.58 \pm 191.7 \mathrm{~mm}^{3}\right)$. In addition, AZD2014 and BEZ235 treatment did not decrease the white blood cell count (Figure 4D).

\section{Discussion}

This study has demonstrated that the dual mTORC1/2 inhibitor AZD2014 had a better anticancer activity compared to the dual PI3K/mTORC1 inhibitor BEZ235 in the mouse graft model. Particularly, AZD2014 effectively reduces the tumor growth at a concentration of $11.5 \mathrm{mg} / \mathrm{kg}$, which is much less compared to the concentration of BEZ235. The cell-based experiments showed that AZD2014 significantly reduces the 

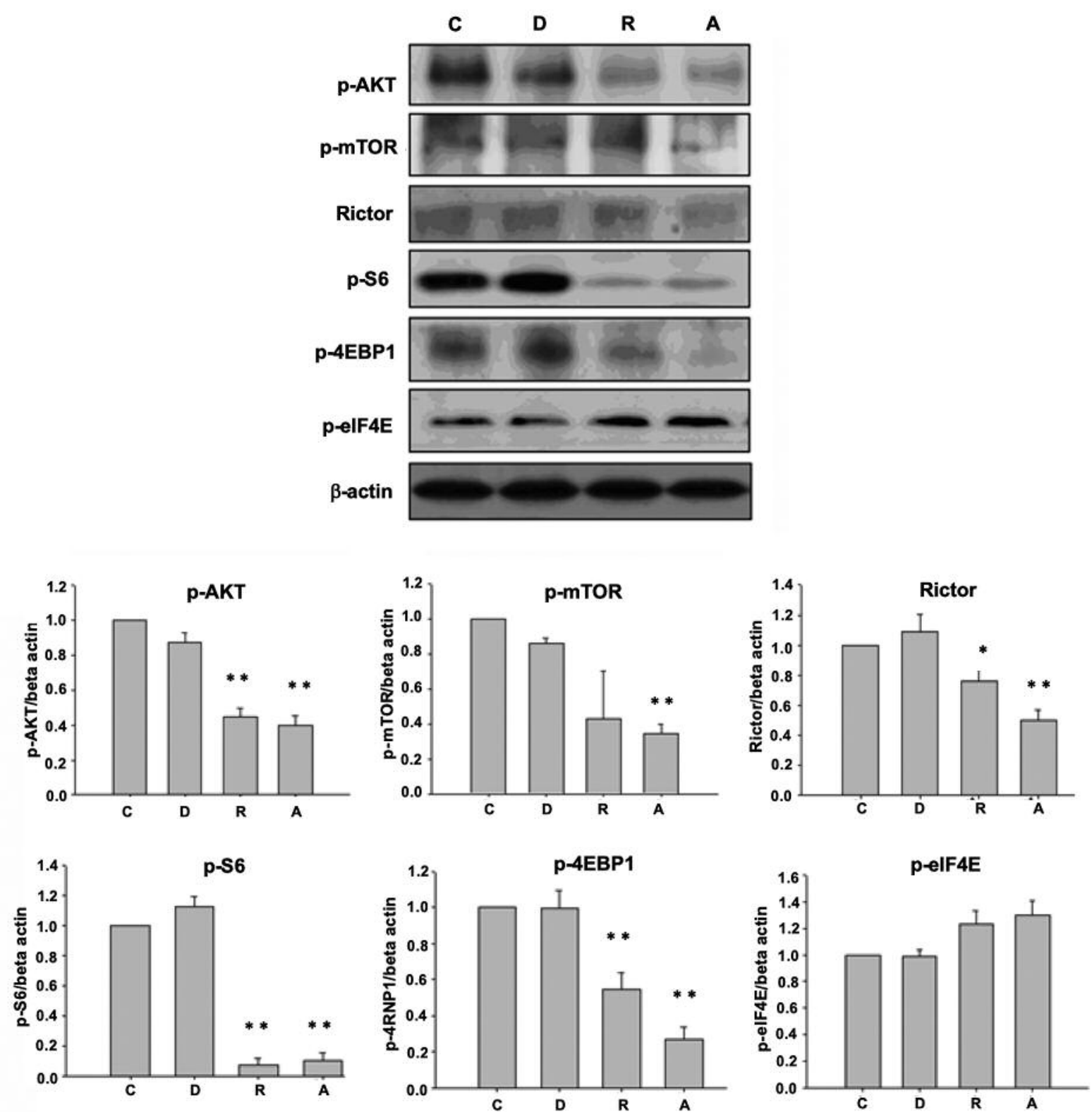

Figure 2. RAD001 and AZD2014 down-regulate the levels of mTORC1 downstream effectors. Western blotting was performed using specific antibodies (left panel). The band intensities of the proteins were quantified using a densitometer following the normalization of $\beta$-actin. L1210 cells were: $i)$ left treated (C) or ii) were treated with DMSO (D), iii) RAD001 (R) at $200 \mathrm{nM}$, or iv) AZD2014 (A) at $100 \mathrm{nM} \mathrm{for} 48$ hours (right panel). The experiments were performed at least three times, with each measurement performed as a triplicate of three independent experiments. Error bars are expressed as a mean $\pm S D(* p<0.05, * * p<0.01)$.

viability of mice L1210 cells and human Toledo cells at the lowest concentration, compared to various inhibitors of the AKT/mTOR signaling, including RAD001, BEZ235, BYL719, and BKM120. Overall, these results indicate that inhibiting the activity of mTORC $1 / 2$ could be used as a potential target for developing inhibitors against CLL.

mTOR inhibition has been indicated as an ideal target for malignant cells (12). Previous research has pointed out that the PI3K/AKT/mTOR signaling pathway may be effective for patients with CLL (13). Therefore, there has been a continuous development of rapamycin and its analogs as immunosuppressant drugs for cancer treatment, including the treatment of CLL $(10,14)$. Another specific mTORC1 inhibitor, temsirolimus, has recently been demonstrated to exhibit stable disease and partial response in several patients with CLL under a phase II trial (15). The rapamycin analog, RAD001, 

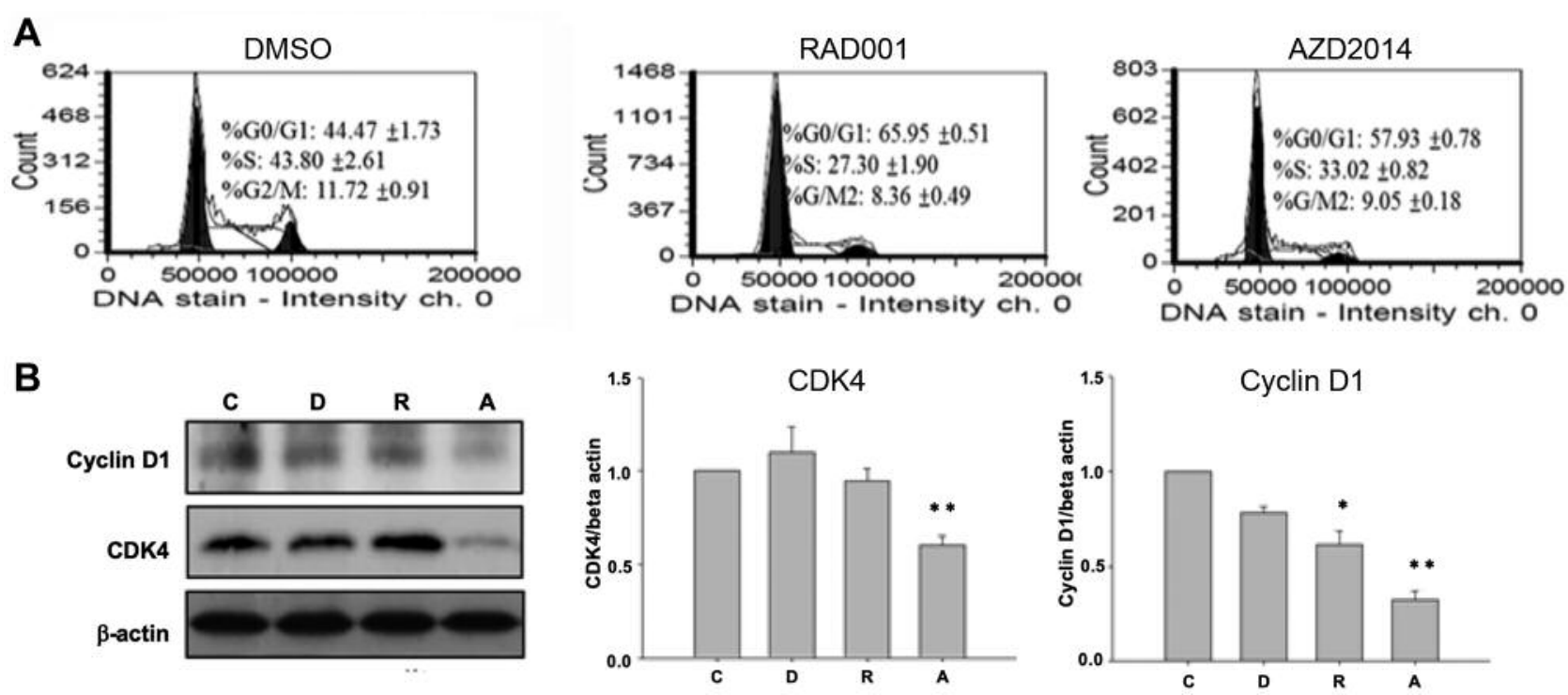

Figure 3. RAD001 and AZD2014 induce cell-cycle arrest by down-regulating the expression of cyclin D1 and CDK4. (A) RAD001 and AZD2014 induced cell-cycle arrest during the $G_{0^{-}} G_{1}$ phase. (B) RAD001 and AZD2014 reduced the expression of cyclin D1 and CDK4. L1210 cells were not treated or treated with DMSO, RAD001, or AZD2014 at 200 and $100 \mathrm{nM}$, respectively, for $48 \mathrm{~h}$. The DAPI-stained cells were analyzed by flow cytometry. The total cell lysate was subjected to western blotting using specific antibodies. The experiments were performed at least three times, with each measurement performed as a triplicate of three independent experiments. Error bars are expressed as a mean $\pm S D(* p<0.05$, **p<0.01).

was included in a phase II clinical trial in patients with advanced B-CLL; however, the trial was stopped because of toxicity concerns (16). This may be due to the ineffective inhibition of mTORC2 or the incomplete blockage of mTORC1, which results in the feedback activation of AKT. Our results reveal that AZD2014 reduces the phosphorylation of mTORC $1 / 2$, leading to a decrease in the activation of AKT/mTOR signaling factors. These results are consistent with the effect of AZD2014 on oral squamous cell carcinoma, where it reduces the activation of mTORC $1 / 2$ and AKT, leading to a decrease in the phosphorylation of the mTOR downstream factors S6K and 4EBP1 (17). In addition, AZD2014 has been used in patients with advanced metastatic breast cancer under a phase I study (18). Therefore, the dual mTORC1/2 inhibitor AZD2014 might be used as a potent inhibitor or a supplement for patients with CLL following evaluation with further preclinical tests.

A previous study reported that the activation of S6K and 4EBP1, the two best characterized factors of mTORC1, regulates protein synthesis by triggering cell proliferation and cell growth (19). Therefore, the phosphorylation of S6K and 4EBP1 is generally used as a biomarker of mTORC1 activity in research studies (20). In our study, we observed that the rapamycin analog RAD001 could not completely reduce the phosphorylated Rictor and 4EBP1 in L1210 cells, suggesting that RAD001 cannot completely block mTORC1 signaling. In contrast, AZD2014 significantly reduced the phosphorylation of 4EBP1 in L1210 cells. A previous study has shown that phosphorylation of 4EBP1 is independent of both mTORC2 and mTORC1 using a knockdown model of mTORC1 or mTORC2 (21). These results demonstrate that AZD2014 blocks the activity of mTOR more completely compared to RAD001 in L1210 cells.

Several studies have reported that mTORC2 regulates cell cycle progression by mediating cyclin D1 stability $(22,23)$. Blockage of mTORC2 activity significantly reduces the translation of cyclin D1 by reducing the recruitment of cyclin D1 mRNA to polysome (24). Moreover, the inhibition of both mTORC 1 and mTORC 2 has been demonstrated to induce cell cycle arrest during the $\mathrm{G}_{0}-\mathrm{G}_{1}$ phase by downregulating the expression of cyclin D1 and CDK4 in hepatocellular carcinoma cell lines (25). Our results show that both RAD001 and AZD2014 significantly block the cell cycle arrest at the G0-G1 phase, whereas RAD001 does not significantly reduce CDK4 expression. These results demonstrate that blockage of mTORC1/2 by AZD2014 may completely decrease CDK4 expression and lead to cell cycle arrest.

In summary, we have demonstrated that the dual mTORC $1 / 2$ inhibitor AZD2014 is a highly potent inhibitor cell growth and proliferation reduction in leukemia L1210 cells. In particular, a more complete inhibition of mTORC1 and CDK4 by AZD2014 compared to the rapamycin analog RAD001, resulting in a more significant reduction in proliferation and cell cycle arrest. These results show that AZD2014 may serve as a potent anticancer agent for patients with CLL and also provide a pathway for further investigation on its effects in leukemia cells. 
A
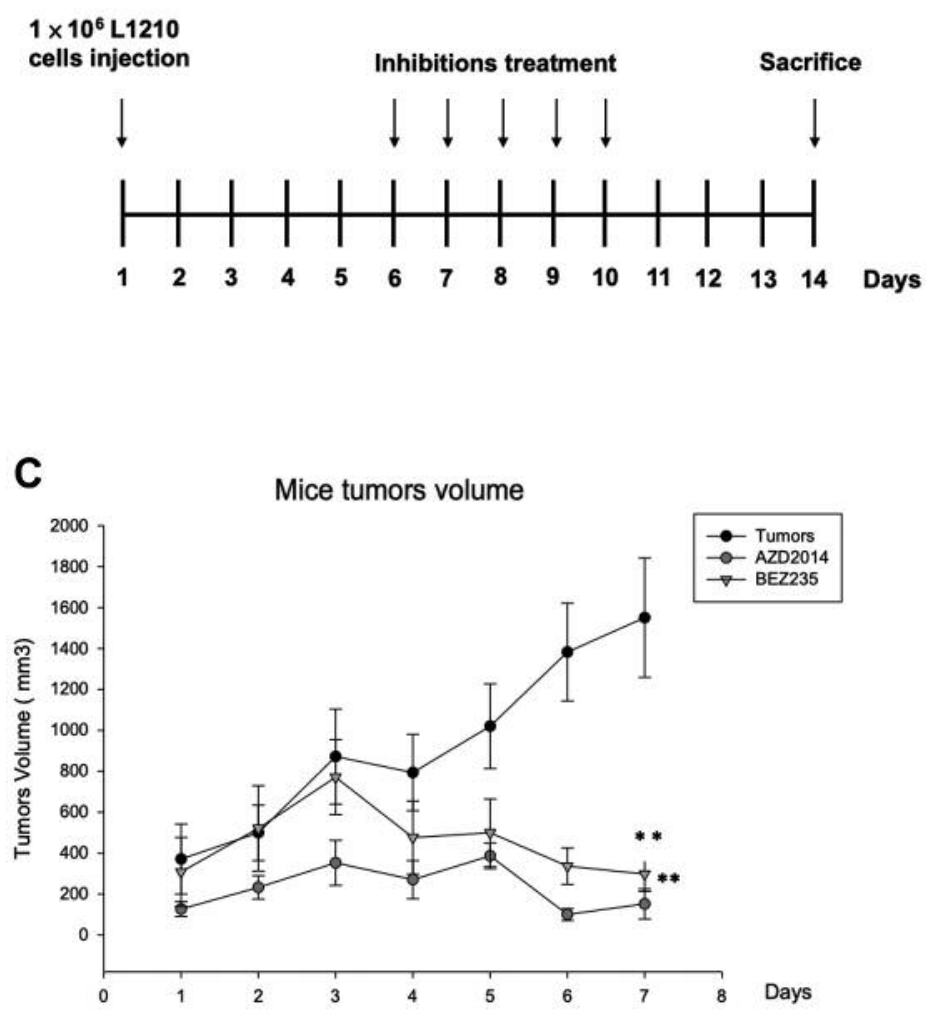

B

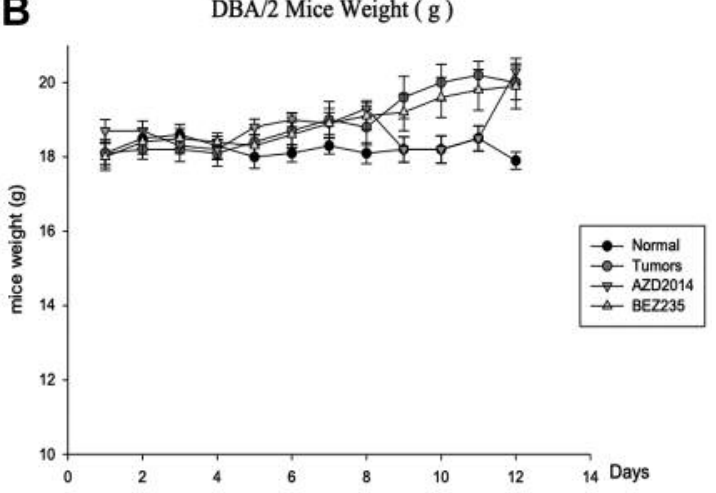

D

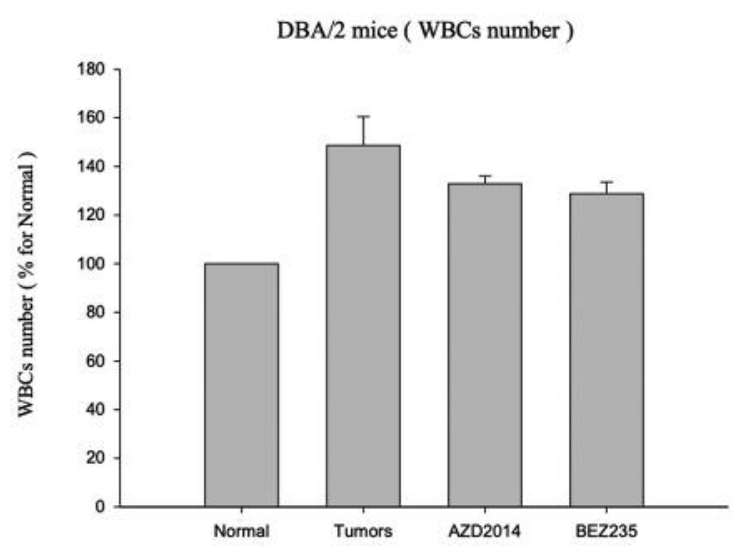

Figure 4. AZD2014 and BEZ235 reduce the tumor growth of L1210 cell grafts in DBA/2 mice. (A) The schematic of the leukemia graft mice model and of the mTOR inhibitor treatment. (B) The body weight of DAB/2 mice was not affected by the treatment with the inhibitors. (C) AZD2014 and BEZ235 inhibited the growth of L1210 cell grafts. The tumor-bearing mice were treated with $11.5 \mathrm{mg} / \mathrm{kg}$ of AZD2014 or $29 \mathrm{mg} / \mathrm{kg}$ of BEZ235 for 5 days. The tumor volume was measured daily for 14 days. (D) The white blood cell count was not affected by the treatment with the inhibitors. Each group comprised six mice $(n=6)$. Error bars are expressed as the mean $\pm S D$ of three independent experiments $\left({ }^{*} p<0.01\right)$.

\section{Conflicts of Interest}

The Authors declare no conflicts of interest.

\section{Authors' Contributions}

Conceived and designed the experiments: YCS and CCY. Performed the experiments and data analysis: HFL, YZL, CCY, TST, and SKU. Wrote the paper: HFL and YCS. All authors reviewed the manuscript.

\section{Acknowledgements}

The Authors are grateful to Novartis and AstraZeneca Pharmaceuticals Corporation for kindly providing the pure compounds. The present study was supported by a grant from the Chi-Mei Medical Center and the Kaohsiung Medical University Research Foundation (108CM-KMU-07) and the Kaohsiung Medical University Hospital (KMUH107-7M13).

\section{References}

1 Chiorazzi N, Rai KR and Ferrarini M: Chronic lymphocytic leukemia. N Engl J Med 352(8): 804-815, 2005. PMID: 15561682. DOI: $10.1056 /$ NEJMra041720

2 Garcia-Munoz R, Galiacho VR and Llorente L: Immunological aspects in chronic lymphocytic leukemia (cll) development. Ann Hematol 91(7): 981-996, 2012. PMID: 22526361. DOI: 10.1007/ s00277-012-1460-Z

3 Herman SE, Gordon AL, Hertlein E, Ramanunni A, Zhang X, Jaglowski S, Flynn J, Jones J, Blum KA, Buggy JJ, Hamdy A, Johnson AJ and Byrd JC: Bruton tyrosine kinase represents a promising therapeutic target for treatment of chronic lymphocytic leukemia and is effectively targeted by pci-32765. Blood 117(23): 6287-6296, 2011. PMID: 21422473. DOI: 10.1182/blood-2011-01-328484

4 Niemann CU, Jones J and Wiestner A: Towards targeted therapy of chronic lymphocytic leukemia. Adv Exp Med Biol 792: 259-291, 2013. PMID: 24014301. DOI: 10.1007/978-1-4614-8051-8_12 
5 Fasolo A and Sessa C: Current and future directions in mammalian target of rapamycin inhibitors development. Expert Opin Investig Drugs 20(3): 381-394, 2011. PMID: 21299441. DOI: $10.1517 / 13543784.2011 .541154$

6 Laplante M and Sabatini DM: Mtor signaling in growth control and disease. Cell 149(2): 274-293, 2012. PMID: 22500797. DOI: 10.1016/j.cell.2012.03.017

7 Chappell WH, Steelman LS, Long JM, Kempf RC, Abrams SL, Franklin RA, Basecke J, Stivala F, Donia M, Fagone P, Malaponte G, Mazzarino MC, Nicoletti F, Libra M, Maksimovic-Ivanic D, Mijatovic S, Montalto G, Cervello M, Laidler P, Milella M, Tafuri A, Bonati A, Evangelisti C, Cocco L, Martelli AM and McCubrey JA: Ras/raf/mek/erk and pi3k/pten/akt/mtor inhibitors: Rationale and importance to inhibiting these pathways in human health. Oncotarget 2(3): 135164, 2011. PMID: 21411864. DOI: 10.18632/oncotarget.240

8 Tenkerian C, Krishnamoorthy J, Mounir Z, Kazimierczak U, Khoutorsky A, Staschke KA, Kristof AS, Wang S, Hatzoglou M and Koromilas AE: Mtorc2 balances akt activation and eif2alpha serine 51 phosphorylation to promote survival under stress. Mol Cancer Res 13(10): 1377-1388, 2015. PMID: 26130148. DOI: 10.1158/1541-7786.MCR-15-0184-T

9 Kim LC, Cook RS and Chen J: Mtorc1 and mtorc2 in cancer and the tumor microenvironment. Oncogene 36(16): 2191-2201, 2017. PMID: 27748764. DOI: 10.1038/onc.2016.363

10 Dancey J: Mtor signaling and drug development in cancer. Nat Rev Clin Oncol 7(4): 209-219, 2010. PMID: 20234352. DOI: 10.1038/nrclinonc.2010.21

11 Fingar DC, Richardson CJ, Tee AR, Cheatham L, Tsou C and Blenis J: Mtor controls cell cycle progression through its cell growth effectors $\mathrm{s} 6 \mathrm{k} 1$ and 4e-bp1/eukaryotic translation initiation factor 4e. Mol Cell Biol 24(1): 200-216, 2004. PMID: 21326806. DOI: $10.1128 / \mathrm{mcb} .24 .1 .200-216.2004$

12 Chan S: Targeting the mammalian target of rapamycin (mtor): A new approach to treating cancer. Br J Cancer 91(8): 1420-1424, 2004. PMID: 15365568. DOI: 10.1038/sj.bjc.6602162

13 Bertacchini J, Heidari N, Mediani L, Capitani S, Shahjahani M, Ahmadzadeh A and Saki N: Targeting pi3k/akt/mtor network for treatment of leukemia. Cell Mol Life Sci 72(12): 2337-2347, 2015. PMID: 25712020. DOI: 10.1007/s00018-015-1867-5

14 Strimpakos AS, Karapanagiotou EM, Saif MW and Syrigos KN: The role of mtor in the management of solid tumors: An overview. Cancer Treat Rev 35(2): 148-159, 2009. PMID: 19013721. DOI: 10.1016/j.ctrv.2008.09.006

15 Fenske TS, Shah NM, Kim KM, Saha S, Zhang C, Baim AE, Farnen JP, Onitilo AA, Blank JH, Ahuja H, Wassenaar T, Qamar R, Mansky P, Traynor AM, Mattison RJ and Kahl BS: A phase 2 study of weekly temsirolimus and bortezomib for relapsed or refractory b-cell non-hodgkin lymphoma: A wisconsin oncology network study. Cancer 121(19): 3465-3471, 2015. PMID: 26079295. DOI: $10.1002 / \mathrm{cncr} .29502$

16 Decker T, Sandherr M, Goetze K, Oelsner M, Ringshausen I and Peschel C: A pilot trial of the mtor (mammalian target of rapamycin) inhibitor rad001 in patients with advanced b-cll. Ann Hematol 88(3): 221-227, 2009. PMID: 18704419. DOI: $10.1007 / \mathrm{s} 00277-008-0582-9$
17 Yu CC, Huang HB, Hung SK, Liao HF, Lee CC, Lin HY, Li SC, Ho HC, Hung CL and Su YC: Azd2014 radiosensitizes oral squamous cell carcinoma by inhibiting akt/mtor axis and inducing $\mathrm{g} 1 / \mathrm{g} 2 / \mathrm{m}$ cell cycle arrest. PLoS One 11(3): e0151942, 2016. PMID: 27031247. DOI: 10.1371/journal.pone.0151942

18 Guichard SM, Curwen J, Bihani T, D'Cruz CM, Yates JW, Grondine M, Howard Z, Davies BR, Bigley G, Klinowska T, Pike KG, Pass M, Chresta CM, Polanska UM, McEwen R, Delpuech O, Green S and Cosulich SC: Azd2014, an inhibitor of mtorc1 and mtorc2, is highly effective in er+ breast cancer when administered using intermittent or continuous schedules. Mol Cancer Ther 14(11): 2508-2518, 2015. PMID: 26358751. DOI: $10.1158 / 1535-7163 . M C T-15-0365$

19 Zent CS, LaPlant BR, Johnston PB, Call TG, Habermann TM, Micallef IN and Witzig TE: The treatment of recurrent/refractory chronic lymphocytic leukemia/small lymphocytic lymphoma (cll) with everolimus results in clinical responses and mobilization of cll cells into the circulation. Cancer 116(9): 2201-2207, 2010. PMID: 20166206. DOI: 10.1002/cncr.25005

$20 \mathrm{Li} \mathrm{S}$, Kong Y, Si L, Chi Z, Cui C, Sheng X and Guo J: Phosphorylation of mtor and s6rp predicts the efficacy of everolimus in patients with metastatic renal cell carcinoma. BMC Cancer 14: 376, 2014. PMID: 24886512. DOI: 10.1186/ 1471-2407-14-376

21 Zhang Y and Zheng XF: Mtor-independent 4e-bp1 phosphorylation is associated with cancer resistance to mtor kinase inhibitors. Cell Cycle 11(3): 594-603, 2012. PMID: 22262166. DOI: $10.4161 / \mathrm{cc} .11 .3 .19096$

22 Koo J, Yue P, Gal AA, Khuri FR and Sun SY: Maintaining glycogen synthase kinase-3 activity is critical for mtor kinase inhibitors to inhibit cancer cell growth. Cancer Res 74(9): 2555-2568, 2014. PMID: 24626091. DOI: 10.1158/0008-5472.CAN-13-2946

23 Carayol N, Vakana E, Sassano A, Kaur S, Goussetis DJ, Glaser H, Druker BJ, Donato NJ, Altman JK, Barr S and Platanias LC: Critical roles for mtorc2- and rapamycin-insensitive mtorc1complexes in growth and survival of bcr-abl-expressing leukemic cells. Proc Natl Acad Sci USA 107(28): 12469-12474, 2010. PMID: 20616057. DOI: 10.1073/pnas.1005114107

24 Altman JK, Sassano A, Kaur S, Glaser H, Kroczynska B, Redig AJ, Russo S, Barr S and Platanias LC: Dual mtorc2/mtorc1 targeting results in potent suppressive effects on acute myeloid leukemia (aml) progenitors. Clin Cancer Res 17(13): 4378-4388, 2011. PMID: 21415215. DOI: 10.1158/1078-0432.CCR-10-2285

25 Chen BW, Chen W, Liang H, Liu H, Liang C, Zhi X, Hu LQ, Yu XZ, Wei T, Ma T, Xue F, Zheng L, Zhao B, Feng XH, Bai XL and Liang TB: Inhibition of mtorc 2 induces cell-cycle arrest and enhances the cytotoxicity of doxorubicin by suppressing mdr1 expression in hcc cells. Mol Cancer Ther 14(8): 1805-1815, 2015. PMID: 26026051. DOI: 10.1158/1535-7163.MCT-15-0029

Received July 10, 2019

Revised July 16, 2019

Accepted July 19, 2019 